\title{
ABT-737 Triggers Caspase-Dependent Inhibition of Platelet Procoagulant Extracellular Vesicle Release during Apoptosis and Secondary Necrosis In Vitro
}

\author{
Hao Wei ${ }^{1}$ Matthew T. Harper ${ }^{1}$ \\ ${ }^{1}$ Department of Pharmacology, University of Cambridge, Cambridge, \\ United Kingdom

\begin{abstract}
Address for correspondence Matthew T. Harper, PhD, Department of Pharmacology, University of Cambridge, Tennis Court Road,

Cambridge CB2 1PD, United Kingdom (e-mail: mth29@cam.ac.uk).
\end{abstract}

Thromb Haemost 2019;119:1665-1674.

\begin{abstract}
Keywords

- platelets

- apoptosis

- microparticles

- extracellular vesicles

- caspase
\end{abstract}

Platelet lifespan is limited by activation of intrinsic apoptosis. Apoptotic platelets are rapidly cleared from the circulation in vivo. ABT-737 triggers platelet apoptosis and is a useful tool for studying this process. However, in vitro experiments lack clearance mechanisms for apoptotic platelets. To determine whether apoptotic platelets progress to secondary necrosis, apoptosis was triggered in human platelets with ABT-737, a $\mathrm{BH} 3$ mimetic. Platelet annexin $\mathrm{V}(\mathrm{AnV})$ binding, release of $\mathrm{AnV}^{+}$extracellular vesicles (EVs), and loss of plasma membrane integrity were monitored by flow cytometry. ABT737 triggered AnV binding, indicating phosphatidylserine exposure, release of $\mathrm{AnV}^{+}$ $\mathrm{EVs}$, and a slow loss of plasma membrane integrity. The latter suggests that apoptotic platelets progress to secondary necrosis in vitro. These responses were dependent on caspase activation and $\mathrm{Ca}^{2+}$ entry. Surprisingly, although intracellular $\mathrm{Ca}^{2+}$ concentration increased, $\mathrm{AnV}^{+} \mathrm{EV}$ release was not dependent on the $\mathrm{Ca}^{2+}$-dependent protease, calpain. On the contrary, ABT-737 downregulated the ability of the $\mathrm{Ca}^{2+}$ ionophore, $\mathrm{A} 23187$, to trigger calpain-dependent release of $\mathrm{AnV}^{+} \mathrm{EV}$ s. This was dependent on caspase activity as, when caspases were inhibited, ABT-737 increased the ability of A23187 to trigger $\mathrm{AnV}^{+}$EV release. These data suggest that apoptotic platelets progress to secondary necrosis unless they are cleared. This may affect the interpretation of ABT-737-triggered signaling in platelets in vitro. $\mathrm{Ca}^{2+}$-dependent $\mathrm{AnV}^{+} \mathrm{EV}$ release is downregulated during apoptosis in a caspase-dependent manner, which may limit the potential consequences of secondary necrotic platelets.

\section{Introduction}

Human platelets circulate for around 10 days before they are cleared. ${ }^{1}$ Although the trigger for platelet clearance has not been fully resolved, intrinsic apoptosis delimits platelet lifespan. ${ }^{2}$ Platelets lacking proapoptotic Bak/Bax have a prolonged circulating half-life in vivo, whereas platelets lacking prosurvival $\mathrm{Bcl}-\mathrm{xL}$ have a very short circulating half-life. ${ }^{3}$ Understanding platelet apoptosis is an important step in understanding disorders of platelet number and function, particularly for understanding the consequences of drugs that trigger platelet apoptosis.

Prosurvival $\mathrm{Bcl}-2$ family proteins, including $\mathrm{Bcl}-\mathrm{xL}$, are upregulated in many cancers and have become attractive targets for anticancer therapy ${ }^{4,5}$ Prosurvival Bcl proteins can be inhibited by BH3 mimetics. One BH3 mimetic, ABT-263 (navitoclax), which inhibits Bcl-2 and Bcl-xL, is associated with thrombocytopenia through activation of intrinsic apoptosis in platelets. ${ }^{6,7}$ This has been avoided through the development of a more selective Bcl2 inhibitor, ABT-199 (venetoclax), which received

February 16, 2019

accepted after revision June 12, 2019
DOI https://doi.org/

10.1055/s-0039-1693694. ISSN 0340-6245. (c) 2019 Georg Thieme Verlag KG Stuttgart · New York
License terms

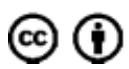


has been approved for treatment of chronic lymphocytic leukemia. ${ }^{8-10}$

BH3 mimetics, such as ABT-737 (an analog of ABT-263), have also become useful tools for investigating apoptosis in platelets in vitro, leading to increased understanding of the signaling that takes place. ${ }^{6,7,11-15}$ ABT-737 induces phosphatidylserine (PS) exposure in platelets in a relatively slow manner-much slower than seen with $\mathrm{Ca}^{2+}$ ionophores or procoagulant combinations of agonists (e.g., thrombin plus collagen-related peptides). ${ }^{7,16}$ The greatest extent of PS exposure is seen after 1 to 3 hours' treatment and so signaling studies often also investigate these late time points (e.g., see Refs. 6, 7, 15, and 17). However, it seems likely that an apoptotic platelet would be cleared within this time under normal circumstances.

Clearance of apoptotic cells in the body is usually rapid. If apoptotic cells are not cleared rapidly they undergo secondary necrosis, which may have proinflammatory or immunogenic consequences. ${ }^{18}$ In vitro, in contrast, the scavengers of apoptotic cells are not present and hence the apoptotic cells will not be rapidly cleared. In this study, we investigated the effect of ABT-737 on platelets in vitro. We demonstrate that although ABT-737 triggers apoptosis in platelets, this progresses to secondary necrosis in vitro. This has important implications for our understanding of platelet apoptosis. Furthermore, our data show that during apoptosis, calpain-dependent extracellular vesicle (EV) release is downregulated. This may be a protective process to limit the potential prothrombotic consequences of platelet necrosis.

\section{Methods}

\section{Washed Platelet Preparation}

Blood was drawn by venepuncture into sodium citrate $(3.2 \% \mathrm{v} / \mathrm{v})$ from healthy, drug-free volunteers, who had given written, informed consent in accordance with the Declaration of Helsinki. Use of human blood for these experiments was approved by the Human Biology Research Ethics Committee, University of Cambridge. Acid citrate dextrose ( $85 \mathrm{mM}$ trisodium citrate, $71 \mathrm{mM}$ citric acid, $111 \mathrm{mM}$ D-glucose) was added (1:7 v/v) and platelet-rich plasma (PRP) separated by centrifugation $(200 \times g, 10$ minutes $)$. Prostaglandin $\mathrm{E}_{1}(100 \mathrm{nM})$ and apyrase (Grade VII; $0.02 \mathrm{U} / \mathrm{mL}$ ) were added to PRP to prevent platelet activation during preparation. Where required, platelets were incubated with either Fluo-4-acetoxymethyl (AM) or calceinAM (both $1 \mu \mathrm{M} ; 10$ minutes). Platelets were pelleted from PRP by centrifugation $(600 \times g, 10$ minutes $)$ and resuspended in HEPES-buffered saline (in mM: 10 HEPES, $135 \mathrm{NaCl}, 3 \mathrm{KCl}, 0.34$ $\mathrm{NaH}_{2} \mathrm{PO}_{4}, 1 \mathrm{MgCl}_{2} \cdot 6 \mathrm{H}_{2} \mathrm{O}$, pH 7.4; supplemented with $0.9 \mathrm{mg} / \mathrm{mL}$ D-glucose) at $5 \times 10^{7}$ platelets $/ \mathrm{mL}$. Platelets were rested $\left(30^{\circ} \mathrm{C}\right.$, 30 minutes) prior to treatment with inhibitors or stimulation. $\mathrm{CaCl}_{2}(2 \mathrm{mM})$ was added immediately prior to simulation.

\section{Flow Cytometry Analysis}

Following stimulation, samples were stained with fluorescein isothiocyanate (FITC)-conjugated annexin V(AnV) (eBioscience, ThermoFisher, United Kingdom), to detect exposed PS (FL1), unless otherwise indicated, and PE-Cy7-conjugated anti-CD41 antibody (eBioscience, ThermoFisher), to distinguish plateletderived events. Samples were analyzed using a BD Accuri C6 flow cytometer. PE-Cy7 fluorescence (FL3) was used to trigger event acquisition. PS-positive platelet-derived EVs were defined as $\mathrm{CD} 41^{+} / \mathrm{AnV}$-positive $\left(\mathrm{AnV}^{+}\right)$events that were smaller than $1 \mu \mathrm{m}$. The $1 \mu \mathrm{m}$ gate was set in forward scatter using $1 \mu \mathrm{m}$ silica beads. ${ }^{19}$ To monitor mitochondrial membrane potential, washed platelets $\left(5 \times 10^{7} / \mathrm{mL}\right)$ were incubated with trimetylrhodamine methyl ester (TMRM; $100 \mu \mathrm{M}, 30^{\circ} \mathrm{C}$, 30 minutes; ThermoFisher) prior to treatment as indicated in the main text. TMRM fluorescence was acquired on FL2.

\section{Immunoblotting}

Platelet proteins were detected in platelet lysates by sodium dodecyl sulfate-polyacrylamide gel electrophoresis and immunoblotting, essentially as described previously. ${ }^{19}$ Anti-talin antibody (clone 8D4; T3287) was from Sigma (Poole, Dorset, United Kingdom), anti-CD41 antibody (ab134131) was from Abcam, and anti-caspase3 (9662), anti-gelsolin (8090), anti-glyceraldehyde 3-phosphate dehydrogenase (2118), anti-extracellular signal-regulated kinases 1 and 2 (4695), and anti-COX IV (4850) were from Cell Signaling Technology (Danvers, Massachusetts, United States), and anti-cytochrome C (sc-13156) was from Santa Cruz Biotechnology (Dallas, Texas, United States). The secondary antibodies used were horseradish peroxidase-conjugated anti-rabbit immunoglobulin $\mathrm{G}(\operatorname{IgG})$ (7074) or anti-mouse IgG (7076; both Cell Signaling Technology).

\section{Data Presentation and Statistical Analysis}

Data are reported as mean \pm standard error of the mean from at least 5 independent platelet preparations, compared using one-way or two-way analysis of variance, as appropriate, in GraphPad Prism v5. Concentration response curves were fitted using a four-parameter logistical equation.

\section{Source of Materials}

All reagents were obtained from Sigma or ThermoFisher unless otherwise stated above. ABT-737 was from Selleck Chemicals.

\section{Results}

\section{ABT-737 Triggers $\mathrm{Ca}^{2+}$-Dependent Apoptosis and Secondary Necrosis}

To investigate platelet apoptosis in vitro, washed human platelets were treated with ABT-737 $(10 \mu \mathrm{M})$ for up to 3 hours. ABT-737 triggered cytochrome $c$ release within 10 minutes (-Supplementary Fig. S1A, available in the online version), which was followed by a slower loss of mitochondrial membrane potential (-Supplementary Fig. S1B, available in the online version). PS exposure was detected by AnV-FITC binding. ABT-737-treated platelets showed two levels of stimulated AnV binding, $A n V^{\text {med }}$ and $\mathrm{AnV}^{\text {high }}$ consistent with previous reports ${ }^{20}$ (-Fig. 1A, B). Coincident with development of $\mathrm{AnV}^{\text {high }}$ platelets, $\mathrm{AnV}^{+} \mathrm{EVs}$ were detected (-Fig. 1A). The $A^{\prime} V^{+}$EVs detected here are 

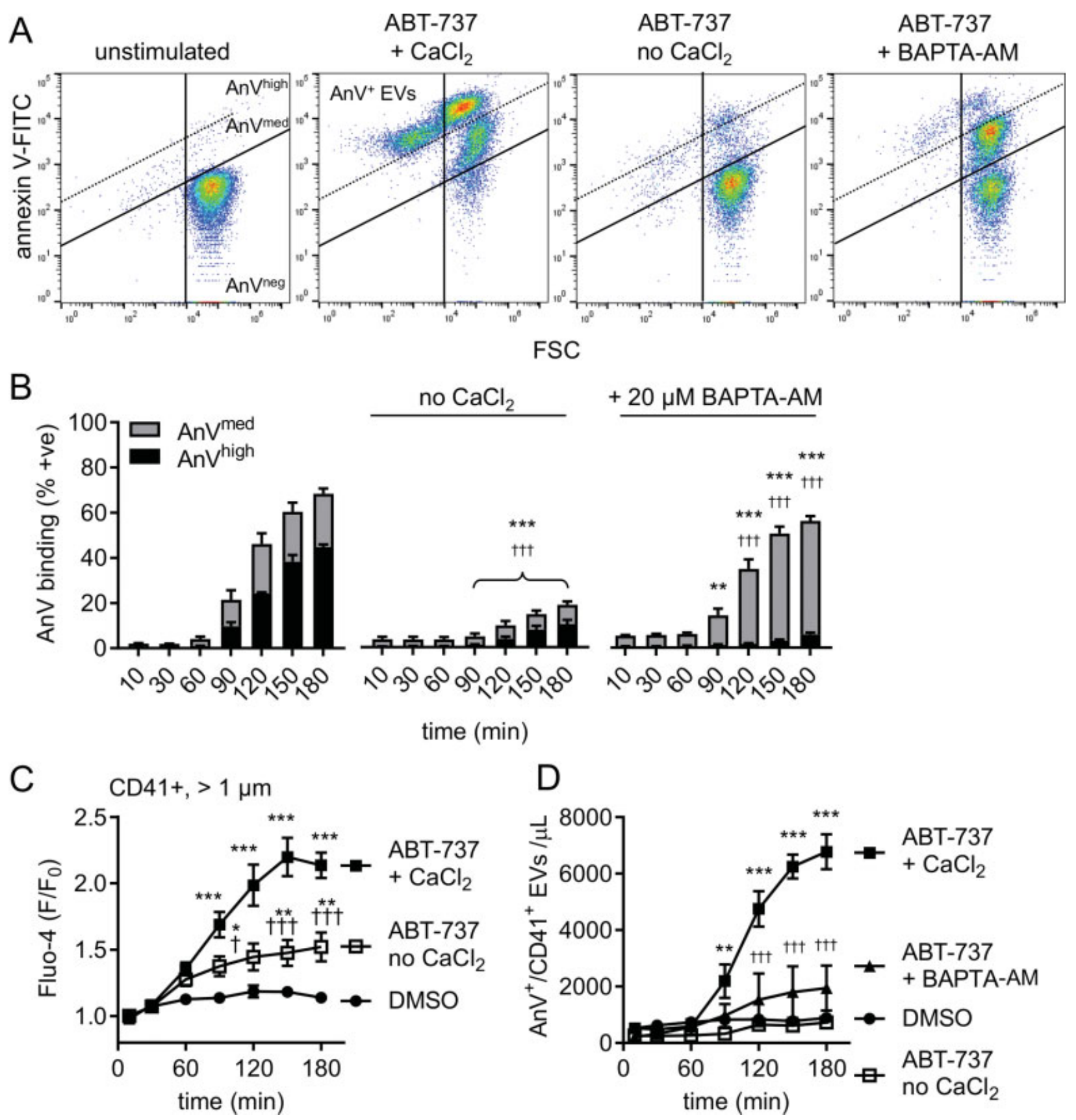

FSC
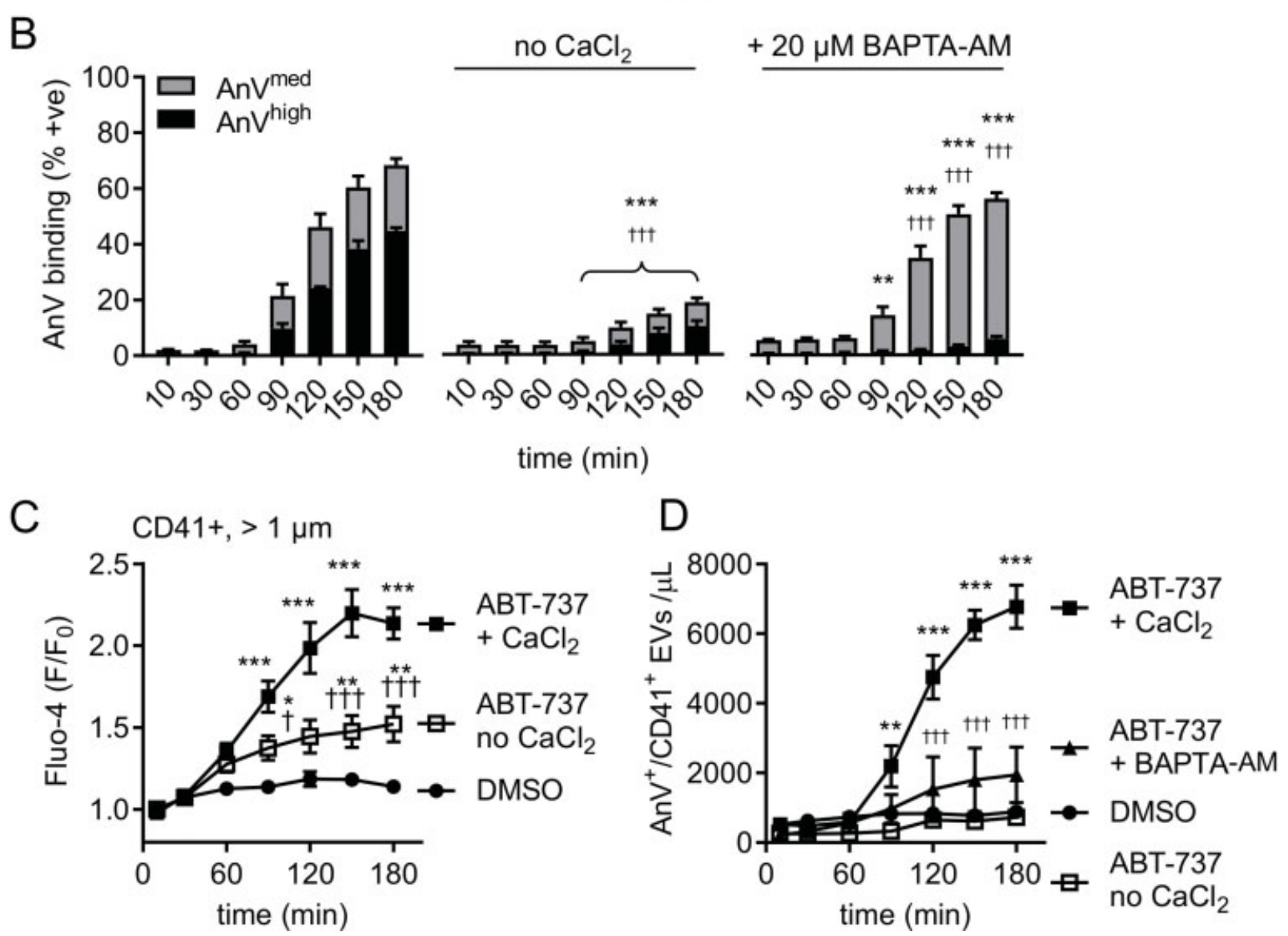

Fig. 1 ABT-737 triggers $\mathrm{Ca}^{2+}$-dependent phosphatidylserine (PS) exposure. (A) Washed human platelets were stimulated with ABT-737 (10 $\mu$ M, 3 hours), after which samples were stained with anti-CD41-PerCP-Cy7, and annexin V (AnV)-fluorescein isothiocyanate (FITC) to detect PS exposure. PerCP-Cy7 fluorescence was used to trigger acquisition of CD41 + events. The panels show density plots of events from low density (blue in online version; black dots in print version) to high density (red in online version; light grey in print version) of forward scatter (FSC-A) and FITC fluorescence. Unstimulated platelets have high FSC-A and low annexin V-FITC binding (AnV ${ }^{\text {neg }}$ ). Stimulation with ABT-737 triggers PS exposure at two levels (AnV'med and AnV high). The vertical line separating left and right was defined by the FSC-A of $1 \mu \mathrm{m}$ silica beads. The density plots are representative of data from 5 different donors. The percentage of platelets with indicated AnV binding in the presence or absence of extracellular $\mathrm{CaCl}_{2}$, or in platelets treated with $20 \mu \mathrm{M}$ BAPTA-AM $(n=5)$ is shown in (B) $\left(n=5 ;{ }^{* *} p<0.01,{ }^{* * *} p<0.001\right.$ for $\mathrm{AnV}^{\text {high }}$ compared with platelets with $\mathrm{CaCl}_{2}$; $\dagger \dagger \dagger p<0.001$ for $\mathrm{AnV}^{\text {med }}$ compared with platelets with $\left.\mathrm{CaCl} 2\right)$. (C) Fluo4-loaded platelets were stimulated with ABT-737 (or dimethyl sulfoxide [DMSO] as vehicle control) for the indicated times. The Fluo-4 fluorescence of platelets (CD41 +, > $1 \mu \mathrm{m}$ ) is shown normalized to the fluorescence of platelets prior to stimulation $\left({ }^{*} p<0.05,{ }^{* *} p<0.01,{ }^{* * *} p<0.001\right.$ compared with DMSO-treated; $t p<0.05$, $\dagger$ t $<0.01$ for no $\mathrm{CaCl}_{2}$ compared with $+\mathrm{CaCl}_{2}$ condition). (D) Number of $\mathrm{AnV}^{+}$extracellular vesicles (EVs) following stimulation with ABT-737 for the indicated times in the presence or absence of extracellular $\mathrm{CaCl}_{2}$ or in BAPTA-AM treated platelets $(n=5)\left({ }^{* *} p<0.01\right.$, ${ }^{* * *} p<0.001$ compared with DMSO-treated; $+\dagger \dagger p<0.001$ for ABT-737 + BAPTA-AM compared with ABT-737 $+\mathrm{CaCl}_{2}$; for clarity, comparison of ABT-737 no CaCl 2 with ABT-737 $+\mathrm{CaCl}_{2}$ or with DMSO-treated is not shown).

likely to be the largest EVs and probably underestimates the total number of EVs present. ${ }^{19}$

ABT-737 also triggered an increase in the intracellular $\mathrm{Ca}^{2+}$ concentration $\left(\left[\mathrm{Ca}^{2+}\right]_{\mathrm{i}}\right)$, which was detected using Fluo-4-load- ed platelets ( - Fig. 1C). Since the earliest time analyzed in this experiment was 10 minutes, the rapid, transient $(<1$ minute) increase in $\left[\mathrm{Ca}^{2+}\right]_{\mathrm{i}}$ that has been previously reported, ${ }^{17}$ was not detected in this assay. The increase in $\left[\mathrm{Ca}^{2+}\right]_{\mathrm{i}}$ was largely 
dependent on the presence of extracellular $\mathrm{Ca}^{2+}$, indicated that it is largely due to $\mathrm{Ca}^{2+}$ entry. $\mathrm{Ca}^{2+}$ entry was required for ABT737-triggered PS exposure and release of $\mathrm{AnV}^{+} \mathrm{EVs}$. AnV binding was significantly inhibited in the absence of extracellular $\mathrm{Ca}^{2+}$ (- Fig. 1B), as previously reported. ${ }^{20}$ In addition, release of $\mathrm{AnV}^{+}$EVs was also abolished (-Fig. 1D). Notably, $\mathrm{CaCl}_{2}$ was present in the AnV staining buffer, as it is required for AnV binding to PS. In control experiments, AnV readily bound to heat-killed platelets when $\mathrm{Ca}^{2+}$ was absent during the treatment and only present in the staining buffer (see - Fig. 2A, for an example).

In contrast, chelation of intracellular $\mathrm{Ca}^{2+}$ by treating platelets with BAPTA-AM $(20 \mu \mathrm{M})$ had less effect. The total percentage of $\mathrm{AnV}^{+}$platelets was not affected. Instead, the percentage of $A n V^{\text {high }}$ platelets was reduced (at 180 minutes, $5.9 \pm 0.8 \%$ were $A^{\text {high }}$, compared with $44.7 \pm 1.1 \% ; n=5$; $p<0.001$ ) and the percentage of $\mathrm{AnV}^{\text {med }}$ platelets correspondingly increased (at 180 minutes, $50.3 \pm 2.2 \%$ were $\mathrm{AnV}^{\mathrm{med}}$, compared with $23.6 \pm 2.4 \% ; n=5, p<0.001$ ), so that the total percentage of $\mathrm{AnV}^{+}$platelets was only slightly reduced $(56.2 \pm 2.3 \%$ in BAPTA-loaded platelets, compared with $68.3 \pm 3.0 \% ; n=5, p<0.05)$. Release of $A^{+} V^{+}$EVs was abolished ( - Fig. 1D), suggesting that their release is linked to the development of $A n V^{\text {high }}$ platelets, and not $A n V^{\text {med }}$ platelets.
Furthermore, some platelets lost plasma membrane integrity, as detected by loss of calcein fluorescence ( - Fig. 2A), indicating that these platelets had progressed to secondary necrosis. Following 3 hours' treatment with ABT-737, $31.1 \pm 1.3 \%$ (-Fig. 2B; $n=5$ ) of platelets had lost plasma membrane integrity. In contrast, following 3 hours' treatment with the solvent, dimethyl sulfoxide (DMSO), only $1.4 \pm 0.1 \%$ of platelets had lost plasma membrane integrity (not shown in - Fig. 2B for clarity). Absence of extracellular $\mathrm{Ca}^{2+}$ prevented secondary necrosis, with only $1.6 \pm 0.2 \%$ (-Fig. 2B; $n=5$ ) of platelets losing plasma membrane integrity. Similarly, BATPA-loading also prevented secondary necrosis, with only $6.0 \pm 0.4 \%$ ( - Fig. $2 \mathrm{~B} ; n=5$ ) of platelets losing plasma membrane integrity. Together, these data show that ABT-737 triggers secondary necrosis in a $\mathrm{Ca}^{2+}$ dependent manner.

\section{ABT-737-Induced Platelet Apoptosis is Caspase- Dependent Whereas Secondary Necrosis Requires Calpain}

Cell death mechanisms often require intracellular proteases. ${ }^{21}$ ABT-737 triggered cleavage of caspase- 3 and its substrate, gelsolin (-Supplementary Fig. S2, available in the online version). Platelet AnV binding, $\mathrm{AnV}^{+} \mathrm{EV}$ release, and loss of calcein fluorescence were inhibited by the pan-caspase
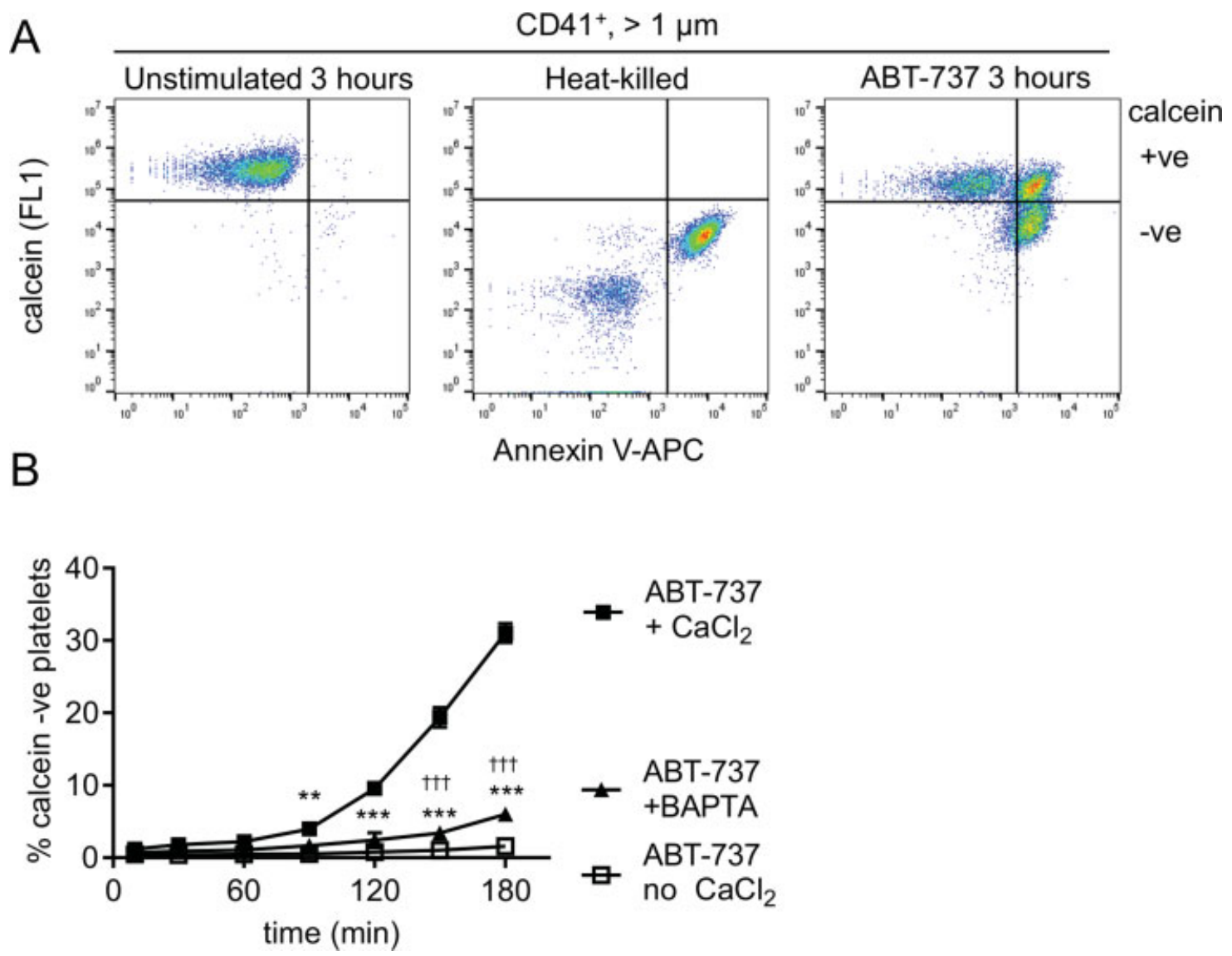

Fig. 2 ABT-737 treatment leads to secondary necrosis. (A) Platelets were loaded with calcein, treated as indicated and stained with anti-CD41PerCP-Cy7, and annexin V-APC. Density plots are gated on platelets (CD41 , > $1 \mu \mathrm{m}$ ) and are representative of 5 independent experiments. The percentage of calcein negative (-ve) platelets is shown in (B) ( $n=5 ;{ }^{* * *} p<0.01$ for $\mathrm{CaCl}_{2} \mathrm{vs}$ no $\mathrm{CaCl}_{2}$; †††p $<0.001$ for CaCl 2 vs. $\mathrm{BAPTA}$. 


\section{A}
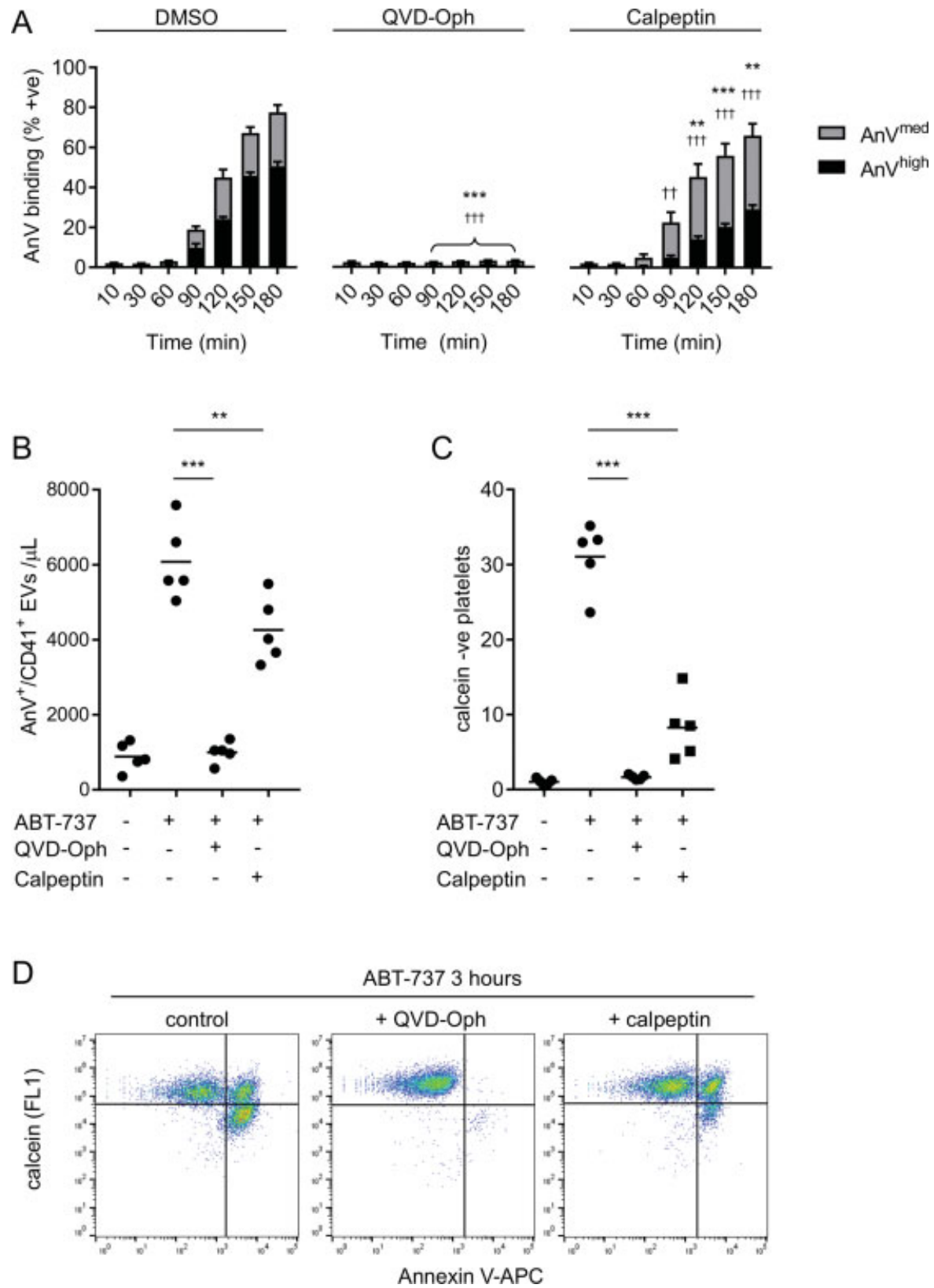

Fig. 3 ABT-737-induced platelet apoptosis is caspase-dependent whereas secondary necrosis requires calpain. (A, B) Platelets were treated with the caspase inhibitor, Q-VD-Oph $(50 \mu \mathrm{M})$, the calpain inhibitor, calpeptin $(140 \mu \mathrm{M})$, or the vehicle (dimethyl sulfoxide [DMSO]) prior to stimulation with ABT-737 for the indicated times. The percentage of platelets with the indicated level of annexin $\mathrm{V}(\mathrm{AnV})$ binding is shown in $(\mathrm{A})\left(n=5 ;{ }^{* *} p<0.05\right.$, ${ }^{* * *} p<0.01$ for AnV high compared with DMSO-treated platelets; $\dagger \dagger p<0.01$, $\dagger \dagger \dagger p<0.001$ for AnVmed compared with DMSO-treated platelets). The number of AnV ${ }^{+}$extracellular vesicles (EVs) detected is shown in (B) $\left(n=5 ;{ }^{* *} p<0.01,{ }^{* * *} p<0.001\right.$ compared with ABT-737-treated platelets). (C, D) Calcein-loaded platelets were treated with Q-VD-Oph or calpeptin then with ABT-737. The mean percentage of calcein -ve platelets is shown in (C), and representative density plots are shown in (D) ( $n=5 ;{ }^{* * *} p<0.01$ compared with ABT-737-treated platelets).

inhibitor, QVD-Oph (50 $\mu \mathrm{M}$; - Fig. 3). QVD-Oph did not inhibit cytochrome $c$ release from mitochondria, consistent with this being upstream of caspase activation during intrinsic apoptosis (-Supplementary Fig. S1A, available in the online version) but did prevent the loss of mitochondrial membrane potential (-Supplementary Fig. S1B, available in the online version). In contrast, the $\mathrm{Ca}^{2+}$-dependent protease, calpain, had a more restricted role in these responses. Calpeptin $(140 \mu \mathrm{M})$, a calpain inhibitor, partially inhibited $A n V^{\text {high }}$ binding, with a corresponding increase in $\mathrm{AnV}^{\text {med }}$ binding (-Fig. 3A). Calpeptin did not significantly affect the loss of mitochondrial mem- brane potential (-Supplementary Fig. S1B, available in the online version). Although calpain activation is required for release of $\mathrm{AnV}^{+}$EVs in response to $\mathrm{Ca}^{2+}$ ionophores or prothrombotic agonists, ${ }^{19,22,23}$ calpeptin had a statistically significant, but small, inhibition of $\mathrm{AnV}^{+} \mathrm{EVs}$ in response to ABT-737 ( - Fig. 3B). Thus, calpain has a relatively restricted role in the release of these $\mathrm{AnV}^{+} \mathrm{EVs}$.

Interestingly, calpeptin inhibited the loss of calcein fluorescence in ABT-737-treated platelets ( - Fig. 3C, D), indicating that calpain activation in apoptotic platelets leads to loss of plasma membrane integrity. In addition, the percentage of AnV ${ }^{\text {high }}$ 
platelets was partially reduced $(29.2 \pm 2.1 \%$ in calpeptintreated platelets, compared with $50.5 \pm 2.3 \%$ in DMSO-treated platelets; $n=5 ; p<0.01 ;-$ Fig. 3A). Some of these AnV ${ }^{\text {high }}$ platelets may in fact be secondary necrotic platelets with a compromised plasma membrane.

\section{Calpain-Dependent Release of $\mathrm{AnV}^{+}$EVs is Downregulated during Apoptosis}

It was surprising that calpain is only weakly involved in ABT-737-triggered release of $\mathrm{AnV}^{+} \mathrm{EVs}$, since calpain is required for release of $\mathrm{AnV}^{+} \mathrm{EVs}$ in response to $\mathrm{Ca}^{2+}$ ionophores or procoagulant agonists. ${ }^{19,23}$ We hypothesized that ABT-737 treatment might somehow reduce the ability of calpain to promote release of $A n V+E V s$. To investigate this further, platelets were treated with ABT-737 for 3 hours in the absence of extracellular $\mathrm{Ca}^{2+}$ (as very few $\mathrm{AnV}^{+}$EVs are released under these conditions). The platelets were then stimulated with the $\mathrm{Ca}^{2+}$ ionophore, $\mathrm{A} 23187$ $(10 \mu \mathrm{M})$, for 10 minutes in the presence of extracellular $\mathrm{Ca}^{2+}$, to trigger a large increase in intracellular $\mathrm{Ca}^{2+}$ concentration (-Fig. 4A) $\mathrm{A} \mathrm{Ca}^{2+}$ ionophore was used rather than physiological agonists that also trigger $\mathrm{AnV}^{+} \mathrm{EV}$

A

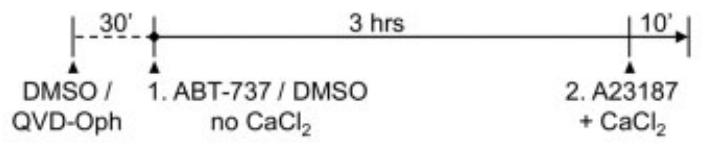

1. DMSO

ABT-737

ABT-737+ QVD-Oph

B
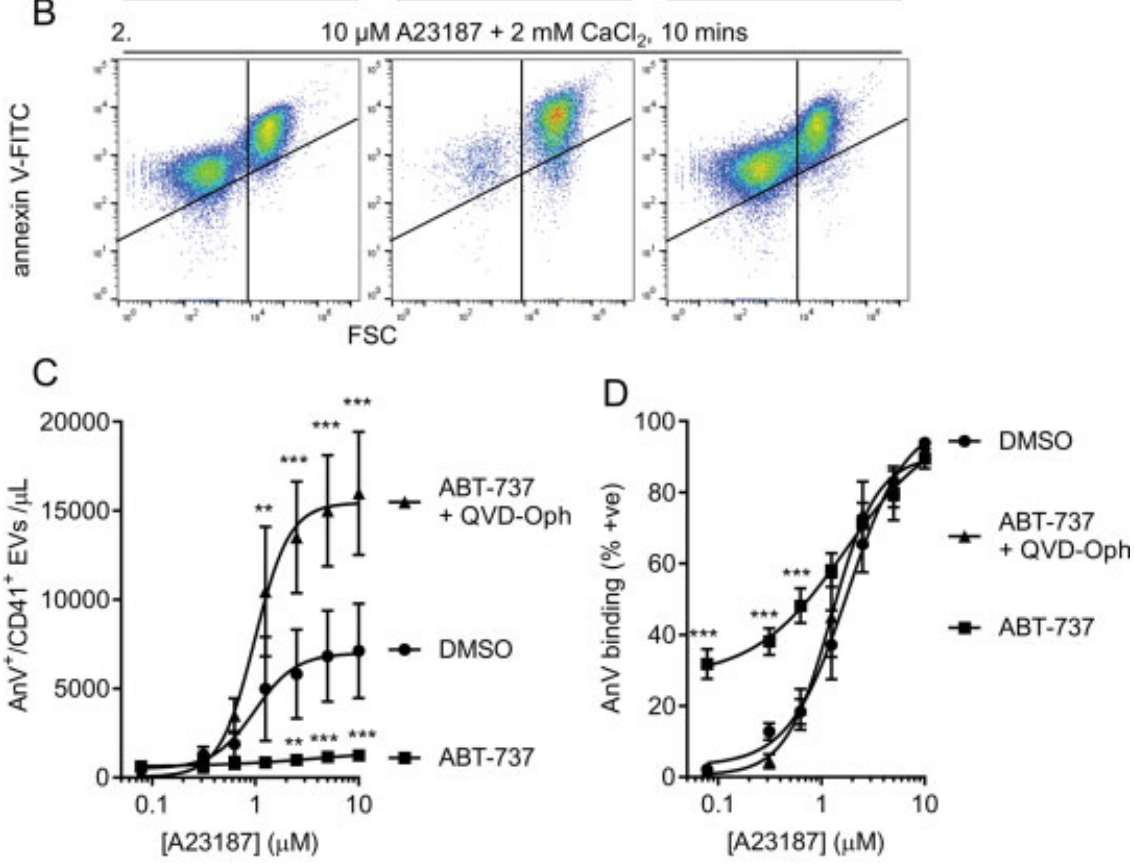

$E$

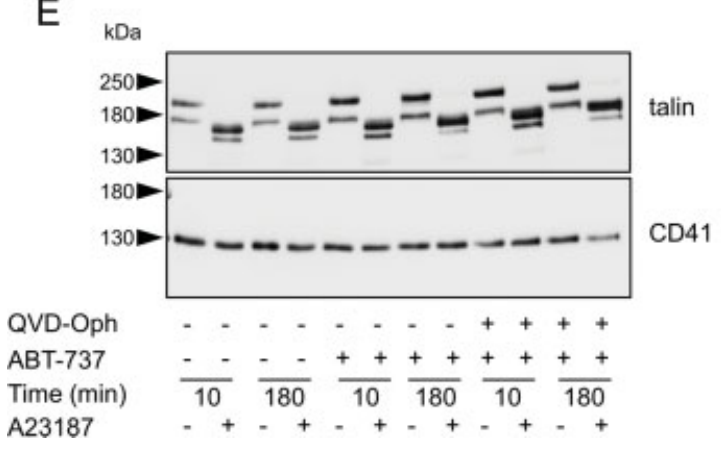

Fig. 4 Calpain-dependent release of annexin $\mathrm{V}$-positive $\left(\mathrm{AnV}^{+}\right)$extracellular vesicle (EVs) is downregulated during apoptosis. (A) Scheme of the experiment. Platelets were treated with Q-VD-Oph (or dimethyl sulfoxide [DMSO] as vehicle control; 30 minutes) prior to ABT-737 (10 $\mu \mathrm{M}$; or DMSO) in the absence of extracellular $\mathrm{CaCl}_{2}$. After 3 hours of $\mathrm{ABT}-737$, platelets were treated with various concentrations of $\mathrm{Ca}^{2+}$ ionophore,

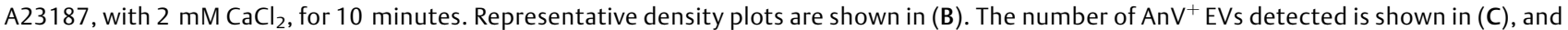
total platelet AnV binding ( $\left.A n V^{m e d}+A n V^{h i g h}\right)$ is shown in (D) $\left(n=5 ;{ }^{* *} p<0.01,{ }^{* * *} p<0.001\right.$ compared with DMSO-treated platelets). In (E),

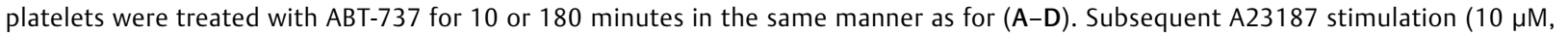
10 minutes) led to cleavage of the calpain substrate, talin, which is unaffected by either ABT-737 or Q-VD-Oph. The blot was stripped and reprobed for CD41, as a loading control, and is representative of 5 independent experiments. 
release (such as thrombin and collagen-related peptide) since ABT-737 treatment disrupts the responses to these agonists, ${ }^{6,7}$ whereas the $\mathrm{Ca}^{2+}$ ionophore can bypass these disruptions.

As expected, $\mathrm{A} 23187 / \mathrm{CaCl}_{2}$ rapidly triggered release of $\mathrm{AnV}^{+}$EVs, whereas ABT-737 triggered very little release of $\mathrm{AnV}^{+}$EVs in the absence of extracellular $\mathrm{Ca}^{2+}$ (-Fig. 1D). However, treatment with ABT-737 inhibited the $\mathrm{AnV}^{+} \mathrm{EV}$ release in response to subsequent stimulation with A23187/ $\mathrm{CaCl}_{2}$ (-Fig. 4B, C). A23187-triggered PS exposure was unaffected (-Fig. 4D). $\mathrm{A} 23187 / \mathrm{CaCl}_{2}$ was still able to activate calpain under these conditions, as shown by cleavage of talin (-Fig. $\mathbf{4 E}$ ). These data suggest that $\mathrm{Ca}^{2+} /$ calpain-dependent release of $\mathrm{AnV}^{+}$EVs are downregulated during apoptosis. The importance of this process is seen when apoptosis was activated but caspases were inhibited (-Fig. 4B, C). These platelets released more $\mathrm{AnV}^{+}$ EVs in response to $\mathrm{A} 23187$, indicating that ABT-737 makes platelets more sensitive to A23187 if caspases are not activated.

\section{Discussion}

Apoptosis of platelets in vivo leads to their rapid clearance from the circulation. ${ }^{3,6}$ Platelet apoptosis can be activated in vitro by ABT-737, making this a useful tool to study the signaling events involved. ABT-737-induced platelet death has been described as apoptosis since ABT-737 triggers cytochrome c release and caspase- 3 activation. ABT-737induced platelet death was not only blocked by caspase inhibitors ${ }^{6,7}$ but also absent in $\mathrm{Bak}^{-1-} \mathrm{Bax}^{-1-}$ mouse plate- lets, ${ }^{3}$ further indicating that it is dependent on the intrinsic apoptosis pathway. However, our data indicate that the responses to ABT-737 in vitro are not a single event, but a temporal sequence of stages leading through to secondary necrosis, each regulated differently by intracellular signaling. These are distinguished by the level of AnV binding, release of $\mathrm{AnV}^{+} \mathrm{EVs}$, and loss of plasma membrane integrity (-Fig. 5). We propose that this last stage represents secondary necrosis.

Two levels of AnV binding were detected, $A n V^{\text {med }}$ and $A n V^{\text {high }}$. The latter is similar to the level seen in platelets stimulated with the $\mathrm{Ca}^{2+}$ ionophore, A23187, or procoagulant combinations of agonists (e.g., thrombin plus collagenrelated peptides). ${ }^{19}$ van Kruchten et al reported a similar pattern in platelets from healthy donors. ${ }^{20}$ (The "M2" population in their study corresponds to $\mathrm{AnV}^{\text {med }}$ here, and "M3" corresponds to $A \mathrm{nV}^{\text {high }}$ here.) One important observation from their study was that when platelets from a patient with Scott syndrome were treated with ABT-737, most $\mathrm{AnV}^{+}$platelets were $\mathrm{AnV}^{\text {med }}$, with few $A n V^{\text {high }}$ platelets. Similarly, in ABT-737-treated platelets from Tmem $16 f^{-1-}$ mice, most $\mathrm{AnV}^{+}$platelets were $\mathrm{AnV}^{\text {med }}$ rather than AnV ${ }^{\text {high }}{ }^{24}$ This suggests that the higher level of PS exposure is due to activation of the $\mathrm{Ca}^{2+}$-dependent phospholipid scramblase, TMEM16F, which is defective in Scott syndrome. ${ }^{25}$ TMEM16F is not responsible for the lower level of PS exposure $\left(\mathrm{AnV}^{\mathrm{med}}\right)$. Another scramblase may be involved, such as the caspase-activated scramblase, $\mathrm{XKR} 8,{ }^{26}$ although this has yet to be reported in platelets.

TMEM16F may be activated by the slow rise in $\left[\mathrm{Ca}^{2+}\right]_{\mathrm{i}}$ detected in Fluo-4-loaded platelets. ${ }^{7}$ This rise was largely

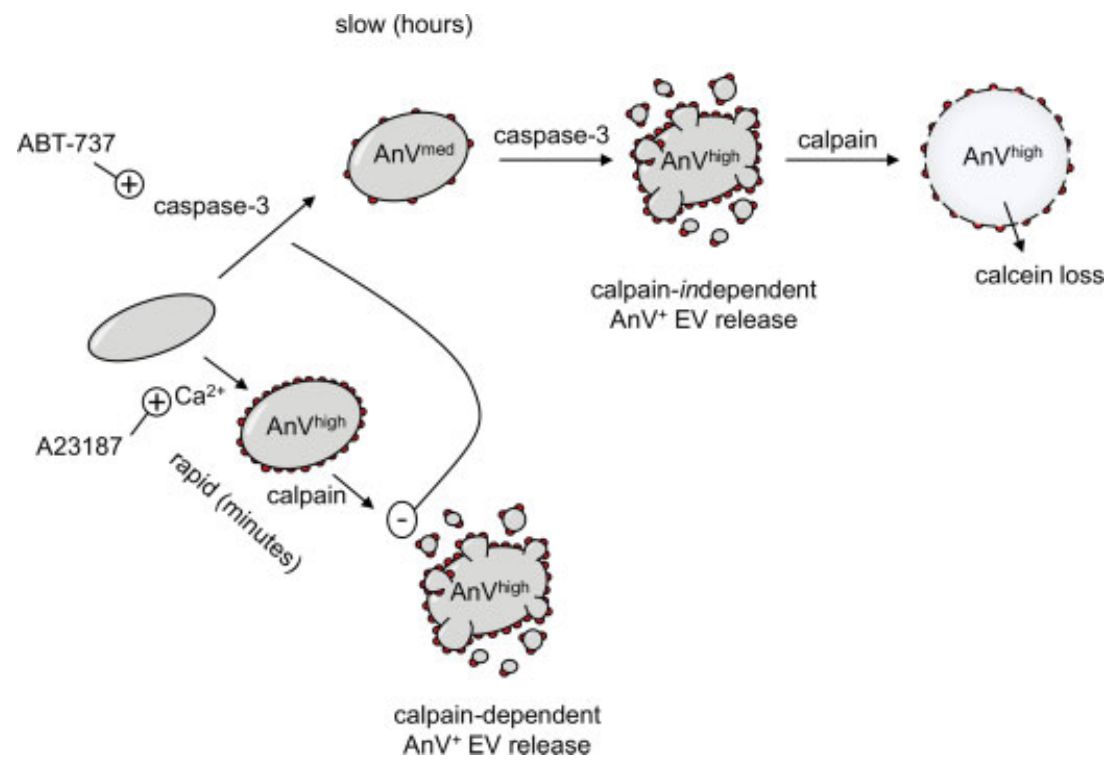

Fig. 5 ABT-737 triggers apoptosis and secondary necrosis. ABT-737 triggers a sequence of responses in platelets that can be distinguished by their sensitivity to different inhibitors. Medium phosphatidylserine (PS) exposure (annexin $V\left[\right.$ AnV ${ }^{\text {med }}$ binding) is triggered in a caspasedependent manner. There is a slow progression to higher PS exposure (AnV high binding), which is blocked by chelation of intracellular Ca ${ }^{2+}$ by BAPTA. These platelets release AnV-positive $\left(\mathrm{AnV}^{+}\right)$extracellular vesicles (EVs) in a calpain-independent manner. Slowly, platelets begin to lose plasma membrane integrity, becoming secondary necrotic. This is dependent on calpain. Caspase activation is required for all these responses and downregulates the rapid release of $\mathrm{AnV}^{+} \mathrm{EVs}$ in response to A23187 and an acute increase in intracellular Ca ${ }^{2+}$. Thus, platelet apoptosis slowly progresses to secondary necrosis if it is not cleared. During the early apoptotic phase, caspases also protect platelets from the effects of prothrombotic stimuli by downregulating the rapid release of prothrombotic EVs. 
due to $\mathrm{Ca}^{2+}$ entry, as it was substantially reduced in the absence of extracellular $\mathrm{Ca}^{2+}$. PS exposure was also dependent on extracellular $\mathrm{Ca}^{2+}$, as absence of extracellular $\mathrm{Ca}^{2+}$ reduced the percentage of $A n V^{\text {high }}$ and $A n V^{\text {med }}$ platelets. Interestingly, chelation of intracellular $\mathrm{Ca}^{2+}$ with BAPTA has less effect. AnV high binding was inhibited, but $A n V^{\text {med }}$ binding was slightly enhanced. A similar result is seen in van Kruchten et al. ${ }^{20}$ This suggests that intracellular BAPTA inhibits the large $\left[\mathrm{Ca}^{2+}\right]_{i}$ signals required to activate TMEM16F but has little effect on the TMEM16F-independent scrambling pathway. In contrast, there is an additional role for extracellular $\mathrm{Ca}^{2+}$. This may be to support $\mathrm{Ca}^{2+}$ entry, or there may be an extracellular site of action. ${ }^{27}$ Whatever the precise roles of $\mathrm{Ca}^{2+}$ in these two pathways of PS exposure, "apoptosis" in response to ABT-737 has distinct stages regulated in different manners.

After prolonged treatment with ABT-737, some platelets lost plasma membrane integrity, suggesting that these platelets have entered secondary necrosis. The progression to secondary necrosis required caspase activation and extracellular and intracellular $\mathrm{Ca}^{2+}$. It also required calpain, a $\mathrm{Ca}^{2+}$-dependent protease (-Fig. 5). Calpain is a major effector of necrotic cell death, and its substrates include cytoskeletal proteins, ion transporters, adhesion molecules, kinases, phosphatases, and phospholipases. ${ }^{28,29}$ The role of calpain in secondary necrosis may account for its small apparent role in PS exposure where the percentage of AnV $V^{\text {high }}$ platelets was partly reduced (- Fig. 3A). Some of these $A n V^{\text {high }}$ platelets may in fact be secondary necrotic platelets with a compromised plasma membrane. This has an important consequence for interpreting in vitro studies with ABT-737, many of which show a similar, partial inhibitions of the percentage of platelets that bind AnV. ${ }^{12,14,15}$ Although these studies are usually interpreted as showing the role of different signaling pathways in platelet apoptosis, it is possible that they are instead showing effects on secondary necrosis.

Surprisingly, calpain has little role in the release of $\mathrm{AnV}^{+}$ EVs during platelet apoptosis. It is surprising since calpain is required for $\mathrm{AnV}^{+} \mathrm{EV}$ release in response to $\mathrm{Ca}^{2+}$ ionophores or procoagulant agonists. ${ }^{30}$ The $\mathrm{AnV}^{+}$EVs appear to derive from the AnV ${ }^{\text {high }}$ platelets, since BAPTA inhibited AnV ${ }^{\text {high }}$ binding and $\mathrm{AnV}^{+} \mathrm{EV}$ release. $\mathrm{AnV}^{\text {high }}$ platelets have a similar level of PS exposure to procoagulant platelets and, as discussed above, are likely to require TMEM16F in the same way as agonist-induced procoagulant platelets. Thus, the $\mathrm{AnV}^{\text {high }}$ platelets and the $\mathrm{AnV}^{+}$EVs generated by ABT737 appear the same as those generated by procoagulant agonists. The difference is that ABT-737 stimulation first triggers caspase activation (with $A n V^{\text {med }}$ binding), and $A n V^{\text {high }}$ binding develops more slowly from these platelets. Our data show that caspase activation inhibits the ability of platelets to subsequently release $\mathrm{AnV}^{+} \mathrm{EVs}$ in response to A23187. It appears that caspases downregulate calpaindependent $\mathrm{AnV}^{+} \mathrm{EV}$ release. This may be important to limit the procoagulant consequences of platelets undergoing apoptosis. ABT-737 triggers mitochondrial outer membrane permeabilization (MOMP) within 10 minutes through Bax/
Bak but independently of subsequent caspase activation (-Supplementary Fig. S1 [available in the online version] and Ref. ${ }^{31}$ ). During apoptosis, MOMP allows mitochondrial cytochrome c release and activation of effector caspases. ${ }^{32}$ MOMP also leads to gradual decline of mitochondrial function. ${ }^{32-34}$ This disruption of mitochondrial function may make platelets more sensitive to procoagulant stimuli, such as $\mathrm{Ca}^{2+}$ ionophores. This was seen when ABT-737 increased the release of $\mathrm{AnV}^{+} \mathrm{EVs}$ in response to $\mathrm{A} 23187$ when caspases were inhibited. Caspases may therefore limit the potential consequences of this by inhibiting $\mathrm{Ca}^{2+} /$ calpaindependent release of $\mathrm{AnV}^{+} \mathrm{EVs}$.

Together these data suggest that induction of apoptosis in platelets triggers a temporal sequence of events. Initially, apoptotic stimuli such as ABT-737 lead to activation of effector caspases. PS is exposed, which acts as an "eat-me" signal for clearance of the apoptotic platelet. ${ }^{35} \mathrm{At}$ the same time, platelets become more sensitive to some procoagulant stimuli that cause an acute rise in $\left[\mathrm{Ca}^{2+}\right]_{\mathrm{i}}$. However, caspases inhibit calpain-dependent release of $\mathrm{AnV}^{+}$EVs, providing a period of reduced capacity to activate platelets, allowing for their safe clearance from the circulation.

However, if the platelet is not cleared (as it will not in vitro owing to lack of scavenger cells), calpain activation leads to loss of plasma membrane integrity and secondary necrosis. Although it is unlikely that platelets normally reach this stage in vivo, rapid induction of apoptosis in many platelets could temporarily overwhelm the capacity to clear them. Circulating $\mathrm{AnV}^{+}$platelets were readily detectable following ABT-737 administration in mice and an approximately $90 \%$ loss of platelet count. ${ }^{6}$ This suggests that if a chemotherapy agent rapidly induced widespread platelet death, the clearance of these dead cells could be temporarily overwhelmed. Impaired clearance and accumulation of secondary necrotic cells has been associated with activation of the adaptive immune system and chronic inflammation. ${ }^{18}$ Necrotic cells release intracellular molecules that act as extracellular signal molecules, known as damage-associated molecular patterns (DAMPs), that activate innate immune cells via receptors including Toll-like receptors. ${ }^{36,37}$ Many DAMPs are now also associated with thrombosis. ${ }^{38-40}$ Although secondary necrotic cells may also release DAMPs, these may have been modified by prior activation of caspases during apoptosis, leading to a different pattern of inflammatory and immunogenic events. ${ }^{41}$ Impaired clearance of apoptotic cells is also associated with the development of autoantibodies against intracellular antigens and chronic inflammation. ${ }^{18,42}$ Although the consequences of platelet secondary necrosis are yet to be determined, a large number of circulating secondary necrotic cells would have the potential to promote acute and chronic inflammation.

In summary, apoptotic platelets progress to secondary necrosis unless they are cleared. Some of the signaling events described in vitro may reflect platelet secondary necrosis, rather than early apoptosis. $\mathrm{AnV}^{+} \mathrm{EV}$ release is downregulated during apoptosis in a caspase-dependent 
manner, which may limit the prothrombotic consequences of cell death.

\section{What is known about this topic?}

- Platelet lifespan is controlled by intrinsic apoptosis.

- BH3 mimetics, such as ABT-737, can trigger platelet apoptosis in vivo and in vitro.

- ABT-737 is commonly used to study the signaling involved in platelet apoptosis in vitro.

\section{What does this paper add?}

- Apoptotic platelets progress to secondary necrosis in vitro as they are not cleared.

- The intracellular signaling involved in the stages of apoptosis and secondary necrosis is different, which affects the interpretation of platelet apoptosis studies in vitro.

- During platelet apoptosis, caspases downregulate the release of procoagulant extracellular vesicles from platelets in response to stimulation.

\section{Note}

The data are available from the corresponding author on reasonable request.

\section{Authors' Contributions}

H.W. performed experiments, analyzed data, and edited the manuscript. M.T.H. designed experiments, wrote, and edited the manuscript

\section{Funding}

None.

\section{Conflict of Interest}

None declared.

\section{Acknowledgments}

This work was supported by an Isaac Newton Trust/Wellcome Trust ISSF/University of Cambridge Joint Research Grant to M.T.H.

\section{References}

1 Lebois M, Josefsson EC. Regulation of platelet lifespan by apoptosis. Platelets 2016;27(06):497-504

2 Kile BT. The role of apoptosis in megakaryocytes and platelets. Br J Haematol 2014;165(02):217-226

3 Mason KD, Carpinelli MR, Fletcher JI, et al. Programmed anuclear cell death delimits platelet life span. Cell 2007;128(06):1173-1186

4 Delbridge AR, Strasser A. The BCL-2 protein family, BH3-mimetics and cancer therapy. Cell Death Differ 2015;22(07):1071-1080

5 Montero J, Letai A. Why do BCL-2 inhibitors work and where should we use them in the clinic? Cell Death Differ 2018;25(01):56-64

6 Schoenwaelder SM, Jarman KE, Gardiner EE, et al. Bcl-xL-inhibitory BH3 mimetics can induce a transient thrombocytopathy that undermines the hemostatic function of platelets. Blood 2011;118 (06):1663-1674
7 Vogler M, Hamali HA, Sun XM, et al. BCL2/BCL-X(L) inhibition induces apoptosis, disrupts cellular calcium homeostasis, and prevents platelet activation. Blood 2011;117(26):7145-7154

8 Souers AJ, Leverson JD, Boghaert ER, et al. ABT-199, a potent and selective BCL-2 inhibitor, achieves antitumor activity while sparing platelets. Nat Med 2013;19(02):202-208

9 Merino D, Kelly GL, Lessene G, Wei AH, Roberts AW, Strasser A. BH3-mimetic drugs: blazing the trail for new cancer medicines. Cancer Cell 2018;34(06):879-891

10 Mihalyova J, Jelinek T, Growkova K, Hrdinka M, Simicek M, Hajek R. Venetoclax: a new wave in hematooncology. Exp Hematol 2018; 61:10-25

11 Kumari S, Chaurasia SN, Kumar K, Dash D. Anti-apoptotic role of sonic hedgehog on blood platelets. Thromb Res 2014;134(06):1311-1315

12 Zhang S, Ye J, Zhang Y, et al. P2Y12 protects platelets from apoptosis via PI3k-dependent Bak/Bax inactivation. J Thromb Haemost 2013;11(01):149-160

13 Winkler J, Rand ML, Schmugge M, Speer O. Omi/HtrA2 and XIAP are components of platelet apoptosis signalling. Thromb Haemost 2013;109(03):532-539

14 Chatterjee M, Borst O, Walker B, et al. Macrophage migration inhibitory factor limits activation-induced apoptosis of platelets via CXCR7-dependent Akt signaling. Circ Res 2014;115(11): 939-949

15 Rukoyatkina N, Mindukshev I, Walter U, Gambaryan S. Dual role of the 38 MAPK/CPLA2 pathway in the regulation of platelet apoptosis induced by ABT-737 and strong platelet agonists. Cell Death Dis 2013; 4:e931

16 Harper MT, Londoño JEC, Quick K, et al. Transient receptor potential channels function as a coincidence signal detector mediating phosphatidylserine exposure. Sci Signal 2013;6(281):ra50

17 Harper MT, Poole AW. Bcl-xL-inhibitory BH3 mimetic ABT-737 depletes platelet calcium stores. Blood 2012;119(18):4337-4338

18 Sachet M, Liang YY, Oehler R. The immune response to secondary necrotic cells. Apoptosis 2017;22(10):1189-1204

19 Wei H, Malcor JM, Harper MT. Lipid rafts are essential for release of phosphatidylserine-exposing extracellular vesicles from platelets. Sci Rep 2018;8(01):9987

20 van Kruchten R, Mattheij NJ, Saunders C, et al. Both TMEM16Fdependent and TMEM16F-independent pathways contribute to phosphatidylserine exposure in platelet apoptosis and platelet activation. Blood 2013;121(10):1850-1857

21 Vanden Berghe T, Linkermann A, Jouan-Lanhouet S, Walczak H, Vandenabeele P. Regulated necrosis: the expanding network of non-apoptotic cell death pathways. Nat Rev Mol Cell Biol 2014;15 (02):135-147

22 Yano Y, Shiba E, Kambayashi J, et al. The effects of calpeptin (a calpain specific inhibitor) on agonist induced microparticle formation from the platelet plasma membrane. Thromb Res 1993;71 (05):385-396

23 Fox JEB, Austin CD, Reynolds CC, Steffen PK. Evidence that agonistinduced activation of calpain causes the shedding of procoagulant-containing microvesicles from the membrane of aggregating platelets. J Biol Chem 1991;266(20):13289-13295

24 Mattheij NJA, Braun A, van Kruchten R, et al. Survival protein anoctamin- 6 controls multiple platelet responses including phospholipid scrambling, swelling, and protein cleavage. FASEB J 2016; 30(02):727-737

25 Suzuki J, Umeda M, Sims PJ, Nagata S. Calcium-dependent phospholipid scrambling by TMEM16F. Nature 2010;468(7325):834-838

26 Suzuki J, Denning DP, Imanishi E, Horvitz HR, Nagata S. Xk-related protein 8 and CED- 8 promote phosphatidylserine exposure in apoptotic cells. Science 2013;341(6144):403-406

27 Hampton MB, Vanags DM, Pörn-Ares MI, Orrenius S. Involvement of extracellular calcium in phosphatidylserine exposure during apoptosis. FEBS Lett 1996;399(03):277-282

28 Zong WX, Thompson CB. Necrotic death as a cell fate. Genes Dev 2006;20(01):1-15 
29 Liu X, Van Vleet T, Schnellmann RG. The role of calpain in oncotic cell death. Annu Rev Pharmacol Toxicol 2004;44:349-370

30 Morel O, Jesel L, Freyssinet JM, Toti F. Cellular mechanisms underlying the formation of circulating microparticles. Arterioscler Thromb Vasc Biol 2011;31(01):15-26

31 Choo H-J, Kholmukhamedov A, Zhou C, Jobe S. Inner mitochondrial membrane disruption links apoptotic and agonist-initiated phosphatidylserine externalization in platelets. Arterioscler Thromb Vasc Biol 2017;37(08):1503-1512

32 Tait SW, Green DR. Mitochondrial regulation of cell death. Cold Spring Harb Perspect Biol 2013;5(09):a008706

33 Lartigue L, Kushnareva Y, Seong Y, Lin H, Faustin B, Newmeyer DD. Caspase-independent mitochondrial cell death results from loss of respiration, not cytotoxic protein release. Mol Biol Cell 2009;20 (23):4871-4884

34 Kushnareva Y, Newmeyer DD. Bioenergetics and cell death. Ann N Y Acad Sci 2010;1201:50-57

35 Ravichandran KS. Find-me and eat-me signals in apoptotic cell clearance: progress and conundrums. J Exp Med 2010;207(09):1807-1817
36 Chen GY, Nuñez G. Sterile inflammation: sensing and reacting to damage. Nat Rev Immunol 2010;10(12):826-837

37 Krysko DV, Agostinis P, Krysko O, et al. Emerging role of damageassociated molecular patterns derived from mitochondria in inflammation. Trends Immunol 2011;32(04):157-164

38 Chen Q, Vogel S, Loughran P, Zuckerbraun BS, Billiar T, Neal MD. Platelet derived HMGB1 regulates thrombosis and organ injury following trauma. Shock 2015;43(06):24

39 Vogel S, Bodenstein R, Chen Q, et al. Platelet-derived HMGB1 is a critical mediator of thrombosis. J Clin Invest 2015;125(12): 4638-4654

40 Wang Y, Fang C, Gao H, et al. Platelet-derived S100 family member myeloid-related protein-14 regulates thrombosis. J Clin Invest 2014;124(05):2160-2171

41 Silva MT. Secondary necrosis: the natural outcome of the complete apoptotic program. FEBS Lett 2010;584(22):4491-4499

42 Mahajan A, Herrmann M, Muñoz LE. Clearance deficiency and cell death pathways: a model for the pathogenesis of SLE. Front Immunol 2016;7:35 